\title{
Hyperkalemia from preoperative non-steroidal anti-inflammatory drugs and angiotensin II receptor blockers in patients with nephropathy
}

\author{
Ho Kyung Song, Yeon Jang, Jin Woo Nam, and Ju-Hyun You \\ Department of Anesthesiology, Inchon St. Mary's Hospital, The Catholic University Medical College, Incheon, Korea
}

Hyperkalemia is sometimes life-threatening and is common in patients with severe renal insufficiency [1]. Some medications such as cyclooxygenase (COX-1, COX-2) inhibitors, angiotensin converting enzyme (ACE) inhibitors, and angiotensin II receptor blockers (ARBs) reduce the elimination of renal potassium resulting in hyperkalemia. Non-steroidal anti-inflammatory drugs (NSAIDs) are often prescribed as pain killers but have known adverse effects on kidney function. Thus, the elderly and patients at risk for diabetic nephropathy, long-lasting hypertension, or dehydration are more susceptible to the adverse effects of these drugs [2].

An episode of decreased renal function can be underestimated when the signs are asymptomatic, transitory, and non-anuric. Recently, ARBs have been prescribed in patients with hypertension to maintain appropriate renal perfusion by decreasing the glomerular filtration burden. ARBs protect renal and cardiac function by lowering oxidative stress, which is associated with chronic changes in the kidneys, heart, and blood vessels. However, ARBs decrease renal blood flow and reduce the elimination of potassium through the kidney under relatively dehydrated conditions [3] and a hypertensive state. Oral administration of ARBs in patients with chronic renal failure has resulted in serum potassium increases of up to $2 \mathrm{mEq} / \mathrm{L}$ during an operation. Although the patient was not suspected to have diabetic nephropathy because of normal creatinine clearance and negative urine protein, a preoperative ACE inhibitor and ARB prescription could lead to hyperkalemia and an abnormal electrocardiogram during an operation [4]. Dehydration has been discussed as a factor in reducing renal blood flow [5]. Thus, chronic administration of ARBs overlapped on possible preoperative dehydration can affect the elimination of potassium resulting in hyperkalemia.

In addition to chronic treatment with an ARB, renal side effects of NSAIDs are relatively common and as prevalent as $5 \%$. NSAIDs affect kidney function in two ways: interstitial nephritis due to an immunological reaction and prerenal failure due to reduced renal plasma flow [2]. Activating the renninangiotensin system to increase glomerular filtration pressure is necessary to maintain sufficient filtration. This physiological mechanism requires sufficient local synthesis of vasodilatory prostaglandins (PGE2, PGI2, PGD2) to counteract the action of angiotensin II on vascular tone. Because NSAIDs inhibit the PG-mediated vasodilation of afferent arterioles and glomerular capillaries, renal blood flow is critically affected in those patients at risk [2].

It is also advisable for anesthesiologists to consider the possibility of renal dysfunction in patients at risk with medication even though there is little chance to identify abnormal signs or abnormal laboratory nephropathy findings. It is more important to perform careful monitoring preoperatively and to evaluate renal function and effective circulatory volume.

\section{References}

1. Putcha N, Allon M. Management of hyperkalemia in dialysis patients. Semin Dial 2007; 20: 431-9.

Corresponding author: Ho Kyung Song, M.D., Ph.D., Department of Anesthesiology, Inchon St. Mary's Hospital, The Catholic University Medical College, 665, Bupyeong-dong, Bupyeong-gu, Incheon 403-720, Korea. Tel: 82-32-280-5410, Fax: 82-32-280-5416, E-mail: genovia@catholic.ac.kr (c) This is an open-access article distributed under the terms of the Creative Commons Attribution Non-Commercial License (http:// creativecommons.org/licenses/by-nc/3.0/), which permits unrestricted non-commercial use, distribution, and reproduction in any medium, provided the original work is properly cited. 
2. Whelton A. Nephrotoxicity of nonsteroidal anti-inflammatory drugs: physiologic foundations and clinical implications. Am J Med 1999; 106: 13S-24S.

3. Halperin ML, Kamel KS. Potassium. Lancet 1998; 352: 135-40.

4. Miyahara Y, Miyazaki T, Tanaka Y, Uchida K, Tamura T, Masuda
$\mathrm{N}$, et al. A case of intraoperative hyperkalemia induced with administration of an angiotensin II receptor antagonist (AIIA) and intake of dried persimmons. Masui 2004; 53: 543-6.

5. Suzuki S, Kashiwagi G, Nakasone Y, Tomioka A, Saito S. Case of unexpected intraoperative hyperkalemia. Masui 2009; 58: 1014-6. 